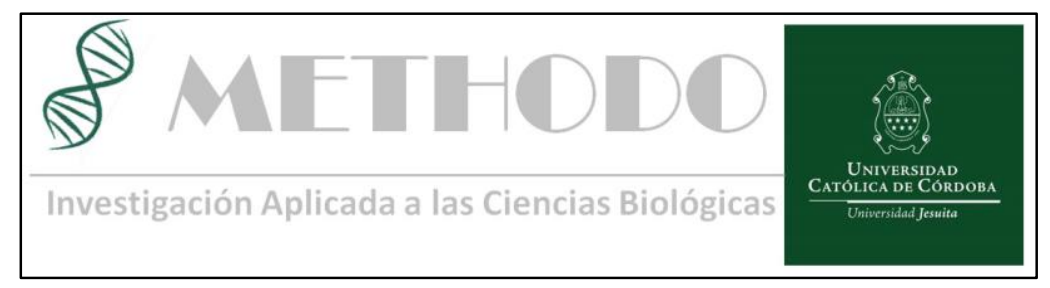

ARTICULO ORIGINAL Methodo 2018;3(1): 06-11 DOI: $10.22529 / \mathrm{me} .2018 .3(1) 03$

\title{
Correlación diagnostica entre fibronasolaringoscopía y video endoscopia digestiva alta en pacientes con reflujo gastroesofágico y reflujo faringolaríngeo.
}

\section{Diagnostic correlation between fibronasolaringoscopy and upper gastrointestinal endoscopy video in patients with gastroesophagic reflux and faringolaryngeal reflux.}

\author{
Torres López $\mathrm{D}^{1}$, Cortadi D ${ }^{1}$, Romero Orellano $\mathrm{F}^{1}$.
}

\begin{abstract}
Resumen: El reflujo faringolaríngeo. Se origina por el flujo retrógrado de contenido gástrico hacia la faringe, pero existen factores, capaces de perpetuarlo: disfunción del esfínter esofágico, tiempo de exposición y sensibilidad del tejido al material refluido, estos elementos permiten considerar al reflujo faringolaríngeo como una entidad diferente del reflujo gastroesofágico.

Objetivo: Encontrar una correlación entre los signos endoscópicos de pacientes con síntomas por reflujo gastroesofágico y reflujo faringolaringeo a través de los hallazgos de la fibrolaringoscopía flexible y video endoscopia digestiva alta. Además de identificar la distribución epidemiológica de los pacientes participantes.

Material y métodos: Estudio observacional, retrospectivo y analítico. Se incluyeron pacientes que consultaron al servicio de Otorrinolaringología y Gastroenterología de la Clínica Universitaria Reina Fabiola Córdoba Argentina, por sintomatología de reflujo faringolaríngeo y gastroesofágico. Entre mayo/2016 y mayo/2017. Los hallazgos de reflujo faringolaríngeo por fibrolaringoscopía flexible y de esofagitis por video endoscopia digestiva alta de cada paciente se compararon con la prueba de Chi cuadrado. Se consideró significativo un valor de $\mathrm{p} \leq 0.05$

Resultados: Se incluyeron 49 pacientes entre 7 y 80 años; de éstos, el 45\% son de género masculino, y $55 \%$ femenino.

El 100\% de los pacientes presentaron criterios fibrolaringoscópicos diagnósticos de reflujo faringolaríngeo, por el contrario, la video endoscopia digestiva alta mostró que solo el 55\% de los pacientes tenían signos de esofagitis $(\mathrm{p}=0.24)$.

Conclusiones: En este estudio, no se observó una correlación diagnóstica entre los signos de la fibrolaringoscopía y video endoscopia digestiva alta en relación al diagnóstico de reflujo faringolaríngeo y esofagitis. No se encontró una diferencia entre ambos géneros y los signos de reflujo faringolaríngeo y esofagitis. La frecuencia de las manifestaciones otorrinolaringológicas del reflujo faringolaríngeo se relaciona directamente con el incremento de la edad; sobre todo, la cuarta década de la vida.

Palabras claves: reflujo faringolaríngeo, reflujo gastroesofágico, esofagitis, laringitis.
\end{abstract}

\begin{abstract}
Pharyngolaryngeal reflux its etiology, the retrograde flow of gastric contents towards the pharynx would be the origin, but there are factors, capable of perpetuating it: dysfunction of the esophageal sphincter, time of exposure and sensitivity of the tissue to the refluxed material, which allows pharyngolaryngeal reflux to be considered as a different entity from gastroesophageal reflux.
\end{abstract}

Revista Methodo: Investigación Aplicada a las Ciencias Biológicas. Universidad Católica de Córdoba Jacinto Ríos 571 Bo Gral. Paz. X5004FXS. Córdoba. Argentina. Tel.: (54) 3514517299 / Correo: methodo@ucc.edu.ar / Web: methodo.ucc.edu.ar | ARTICULO ORIGINAL Methodo 2018;3(1): 06-11 
Objective: Find a correlation between the endoscopic signs of patients with symptoms due to gastroesophagic reflux and faringolaryngeal reflux through the findings of fiber-optic laryngoscopy and upper gastrointestinal endoscopy. In addition, identify the epidemiological distribution of the participant's patients

Material and methods: Observational, retrospective and analytical study of patients who have consulted the service of Otorhinolaryngology and Gastroenterology of the Reina Fabiola University Clinic, due to symptomatology of pharyngolaryngeal reflux and gastroesophageal reflux. Between May/2016 and May/2017. The findings of pharyngolaryngeal reflux by fiber-optic laryngoscopy and esophagitis by upper gastrointestinal endoscopy of each patient were compared with the Chi square test. A value of $\mathrm{p} \leq 0.05$ was considered significant.

Results: We included 49 patients between 7 and 80 years old; $45 \%$ of them were male and $55 \%$ were female. $100 \%$ of the patients had fiber-optic laryngoscopy diagnostic criteria of pharyngolaryngeal reflux. In contrast, upper digestive endoscopy showed that only $55 \%$ of the patients had signs of esophagitis $(\mathrm{p}=0.24)$.

Conclusions: In this study, wasn't observed a diagnostic correlation between the signs of fiber-optic laryngoscopy and upper gastrointestinal endoscopy in relation to the diagnosis of pharyngolaryngeal reflux and esophagitis. We did not find a difference between both genders and signs of esophageal pharyngolaryngeal reflux. The frequency of otorhinolaryngological manifestations of pharyngolaryngeal reflux is directly related to the increase in age.

Key words: pharyngolaryngeal reflux, gastroesophageal reflux, esophagitis, laryngitis.

1. Servicio de Otorrinolaringología, Clínica Universitaria Reina Fabiola, Universidad Católica de Córdoba, Argentina. Correspondencia: Daniel Torres López. Clínica Universitaria Reina Fabiola. Oncativo 1248 -X5004FHP- Córdoba, Argentina. e-mail: dantorr25@ hotmail.com

\section{Introducción}

La enfermedad por reflujo gastroesofágico es un término clínico que se refiere al reflujo gastroesofágico (RGE) excesivo, que causa daño tisular (esofagitis). El reflujo faringolaríngeo (RFL) se refiere al retorno del contenido gástrico hacia la laringofaringe ${ }^{1}$. Aunque se han sugerido varios nombres para las alteraciones laríngeas inducidas por el reflujo, la nomenclatura adoptada por la Academia Americana de Otorrinolaringología y cirugía de Cabeza y Cuello es la de reflujo faringolaríngeo (RFL), nombre de una enfermedad con síntomas, manifestaciones clínicas, diagnóstico y tratamiento específicos ${ }^{1}$.

El RFL está conformado por una serie de síntomas, incluidas la laringitis, disfonía, fatiga vocal, globus, carraspeo excesivo, tos crónica y disfagia leve. Se sabe que es causado por el reflujo del contenido gástrico del estómago y que es una enfermedad que se produce en la parte superior del esófago, que es donde ocurre el $\mathrm{RGE}^{2,3}$. Según Koufman y col. el RFL es una afección gastrointestinal y otorrinolaringológica relacionada pero distinta de la enfermedad por
RGE, cuyas manifestaciones en la cabeza y el cuello son considerablemente diferentes de las manifestaciones por RFL. Este conocimiento mayor acerca del RFL puede ocasionar un sobrediagnóstico, porque los síntomas del RFL no son específicos y porque éstos pueden ser originados por infecciones, abuso vocal, alergias, fumar o abuso de alcohol ${ }^{4-5-6}$.

La importancia del RFL radica en que se presenta en hasta el $10 \%$ de los pacientes que acuden a la consulta otorrinolaringológica. Además, se ha observado que en más del $50 \%$ de los pacientes con disfonía, existen signos de la existencia de una enfermedad por reflujo subyacente ${ }^{5}$.

El diagnóstico de RFL se confirma de tres $\operatorname{modos}^{6}:$ 1) síntomas que responden al tratamiento empírico, 2) lesión mucosa que se observe por vía endoscópica, y 3) existencia del reflujo se demuestre mediante estudios complementarios, como la impedanciometría y la pH-metría esofágicas. En la práctica es común iniciar la supresión ácida con dosis altas de inhibidores de bomba protones (omeprazol) y evaluar la respuesta al tratamiento tres o cuatro meses después. Una pH-metría ambulatoria de 24 horas, de doble balón y simultánea (esofágica 
Torres López D, Cortadi D, Romero Orellano F Correlación diagnostica entre fibronasolaringoscopía y video endoscopia digestiva alta en pacientes con reflujo gastroesofágico y reflujo faringolaríngeo

y faríngea) es el patrón de referencia actual para establecer el diagnóstico de reflujo laringofaríngeo ${ }^{4,5,8,9}$. Sin embargo, este examen dista mucho de ser una prueba ideal la sensibilidad de la pH-metría es sólo del 50 al $80 \%$, aproximadamente $12 \%$ de los pacientes no tolera el procedimiento, las modificaciones en la dieta pueden ocasionar falsos negativos, es un procedimiento costoso, entre otros ${ }^{7}$. Además de los problemas técnicos vinculados con el material y de las condiciones de prueba que restringen un poco al paciente, hay que hacer notar que la pH-metría no sabe reconocer el reflujo alcalino de origen duodenal o cáustico. Por eso, la Academia Americana de Otorrinolaringología y Cirugía de Cabeza y Cuello señala que el diagnóstico de RFL debe establecerse con base en los síntomas y los $\operatorname{signos}^{10,11}$. Por su parte, con la laringoscopia se obtienen resultados altamente sugestivos aunque no patognomónicos- de RFL, como engrosamiento, hiperemia y edema concentrado en la laringe posterior (laringitis posterior) ${ }^{4,6}$. Otros hallazgos que también se han asociado estrechamente con el RFL son el granuloma de contacto con una frecuencia del $65-74 \%$ de los casos confirmados con pH-metría y psudosulcus. En el presente trabajo se compararon los informes de las video endoscopias digestivas altas (VEDA) y fibrolaringoscopías realizadas en pacientes con síntomas por RFL y RGE.

\section{Objetivos}

\section{Objetivo general}

Comparar las lesiones macroscópicas en el epitelio laríngeo y esofágico (laringitis esofagitis) en pacientes con clínica sugestiva de RFL y RGE.

\section{Objetivo específico}

Encontrar una correlación entre los signos endoscópicos de pacientes con síntomas por RGE y RFL a través de los hallazgos de la fibrolaringoscopía flexible y video endoscopia digestiva alta. Identificar la distribución epidemiológica de los pacientes participantes.

\section{Material y método}

Diseño Estudio observacional, retrospectivo, y analítico.

\section{Población - Entorno}

Se incluyeron pacientes que consultaron al Servicio de Otorrinolaringología y Gastroenterología de la Clínica Universitaria Reina Fabiola en Córdoba, Argentina, por sintomatología de RGE y RFL entre mayo de 2016 y mayo de 2017, en los que se hubiera realizado fibrolaringoscopía flexible y VEDA.

Para el diagnóstico de RFL se utilizó la clasificación adaptada de Belafsky ${ }^{15}$ (en anexo tabla 1). Este índice de hallazgos de RFL, cuyo puntaje máximo es 24 , requiere un puntaje de $\geq 7$ para ser considerado altamente sospechoso de RFL. La misma se divide en tres grados de gravedad: leve (puntaje 7-8), moderado (puntaje 9-16), grave ( $\geq 17)$.

Para el diagnóstico de esofagitis y clasificación de su severidad, se utilizó la clasificación endoscópica de Los Ángeles ${ }^{16}$. La misma, estadifica a la esofagitis en cuatro grados. Estos datos se muestran en la tabla 2 (Ver anexo).

Se identificaron las fibrolaringoscopías y VEDA de pacientes con diagnóstico de reflujo esófago faringolaríngeo, y se seleccionaron los estudios con diagnóstico de enfermedades asociadas con manifestaciones otorrinolaringológicas y esofágicas de RGE - RFL.

Se excluyeron los datos de pacientes con enfermedades alérgicas, infecciosas crónicas rinosinusales, abuso vocal, tabaquistas, en tratamiento con Inhibidores de la bomba de protones y abuso de alcohol al momento de la consulta.

Análisis Estadístico Los datos de la revisión de las fibrolaringoscopías y VEDA se registraron directamente en la ficha de registro; posteriormente, la información se procesó por edad, género, hallazgos en fibrolaringoscopía y VEDA, en cuadros de distribución de frecuencia; la información también se sometió a una comprobación estadística realizada mediante la prueba de chi cuadrado, Test $\mathrm{T}$ de student $\mathrm{y}$ Regresión Lineal Simple. Se utilizó el programa estadístico R-MEDIC®

\section{Resultados}

Se incluyeron 49 pacientes entre 7 y 80 años; de éstos, el $55 \%$ eran de género femenino. Los pacientes de género femenino tenían una edad media de 46.8 (DE 12.5) y los pacientes de género masculino una edad media de 43.9 años (DE 19.0) $(\mathrm{p}=0.53)$.

En cuanto a la edad y los hallazgos en la fibrolaringoscopía se presenta una edad media de 45.5 años (DE 15.5) y las lesiones mas graves se registran a mayor edad (Gráfico 1$)$.

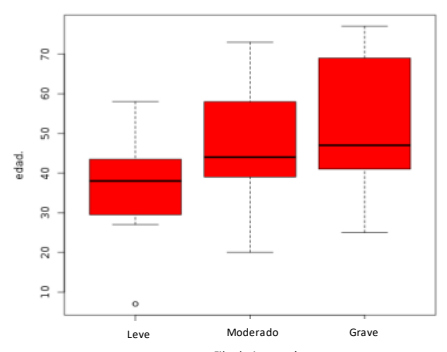

Gráfico 1. Distribucioción de la edad de acuerdo al grado de RFL. 
Torres López D, Cortadi D, Romero Orellano F Correlación diagnostica entre fibronasolaringoscopía y video endoscopia digestiva alta en pacientes con reflujo gastroesofágico y reflujo faringolaríngeo

Observándose una asociación lineal entre edad y la presencia de RFL ( $\mathrm{p}=0.01)$ (Gráfico 2)

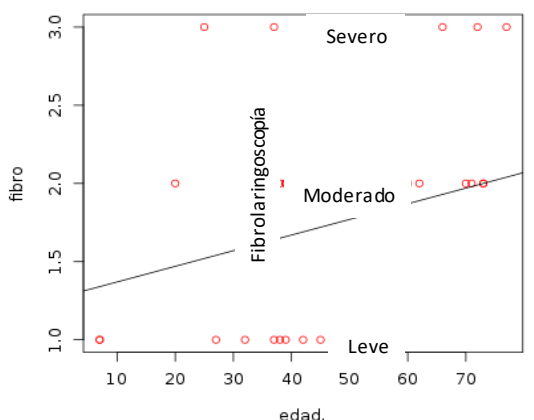

Gráfico 2. Distribución de la edad de acuerdo al grado de RFL.

El $100 \%(\mathrm{~N}=49)$ presentaban por medio de la fibrolaringoscopía criterios diagnósticos de RFL (leve 22\% - moderado 63\% - severo 14\%)

(Gráfico 3) y por medio de VEDA, el 55\% $(\mathrm{N}=27)$ de los pacientes tenían algún grado de

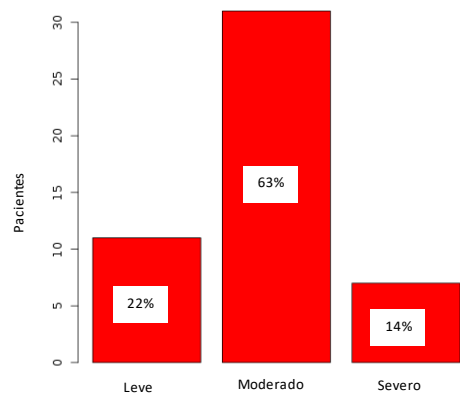

Gráfico 3. Distribución de pacientes de acuerdo al grado de RFL.

esofagitis (grado: A 39\%, B 10\%, C 0\%, D 2\%, Barret 4\%) $(\mathrm{p}=0.24)$ (Gráfico 4).

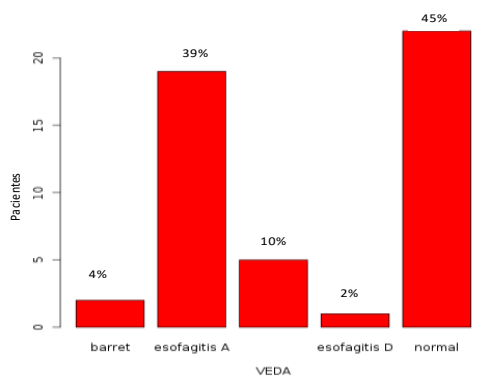

Gráfico 4. Distribución de pacientes de acuerdo al grado de esofagitis por VEDA

Del $100 \%(\mathrm{~N}=49)$ presentaron por medio de la fibrolaringoscopía criterios diagnósticos de RFL y por medio de VEDA, el $45 \%(\mathrm{~N}=22)$ de los pacientes no tenía ningún signo de esofagitis $(\mathrm{p}=$ 0.24) (Tabla 3).

Tabla 3. Correlación de signos en Fibrolaringoscopía y VEDA.

\begin{tabular}{|l|l|l|l|l|l|c|}
\hline & $\begin{array}{l}\text { Barret } \\
\text { N (\%) }\end{array}$ & $\begin{array}{l}\text { esofagitis A } \\
\text { N (\%) }\end{array}$ & $\begin{array}{l}\text { esofagitis B } \\
\text { N (\%) }\end{array}$ & $\begin{array}{l}\text { Esofagitis D } \\
\text { N (\%) }\end{array}$ & $\begin{array}{l}\text { Normal } \\
\text { N (\%) }\end{array}$ & $\begin{array}{l}\text { Valor de } \\
\text { p }\end{array}$ \\
\hline 1 leve & $1(2)$ & $4(8)$ & $1(2)$ & $0(0)$ & $5(10)$ & \multirow{2}{*}{0.24} \\
\hline 2 moderado & $1(2)$ & $9(18)$ & $3(6)$ & $1(2)$ & $17(35)$ & \\
\cline { 1 - 5 } 3 severo & $0(0)$ & $6(12)$ & $1(2)$ & $0(0)$ & $0(0)$ & \\
\hline
\end{tabular}

\section{Discusión}

En este estudio de pacientes con sintomatología de RFL, los hallazgos de la fibrolaringoscopía no tuvieron una asociación estadísticamente significativa con los hallazgos de la VEDA, ya que en aproximadamente sólo la mitad de los pacientes con hallazgos por fibrolaringoscopía se pudo observar algún grado de esofagitis por VEDA. Existen pocos datos acerca de la correlación endoscópica de RGE y RFL. En el año 2007, Vavricka y cols, presentaron los resultados de un estudio, a través del cual se evaluó el valor diagnóstico de la presencia de hallazgos sugerentes de RFL en pacientes con RGE. En total, fueron evaluados 1.209 pacientes. El número final de pacientes Incluidos fue de 132 pacientes. Todos ellos presentaban hallazgos compatibles con esofagitis erosiva (12). Los resultados fueron comparados con un grupo control de igual número de pacientes, sin síntomas de RGE con VEDA normal, se encontraron signos de RFL con una frecuencia similar en ambos grupos (44\% vs 37\%). Estos resultados son similares a lo encontrado en el trabajo actual donde no se encontró una correlación entre los hallazgos de la fibrolaringoscopía y la VEDA (13).

Respecto al género, no se encontró diferencia significativa en la frecuencia del RFL y RGE. Lo cual difiere con la literatura científica donde se encuentra una mayor incidencia en el género femenino (13).

Por su parte, se observó una mayor frecuencia de RFL a mayor edad. Esto coincide con los datos publicados por Montes(14), dando cuenta de la asociación de RFL con la edad.

\section{Conclusiones}

En este estudio no se observó una asociación entre los signos de la fibrolaringoscopía y la video endoscopia digestiva alta (VEDA) para RFL y RGE. Las manifestaciones otorrinolaringológicas del RFL se relacionan directamente con el incremento de la edad; por el contrario, la frecuencia de RFL no parece diferir de acuerdo al género. 
Torres López D, Cortadi D, Romero Orellano F Correlación diagnostica entre fibronasolaringoscopía y video endoscopia digestiva alta en pacientes con reflujo gastroesofágico y reflujo faringolaríngeo

\section{Bibliografía}

1. Koufman JA, Aviv JE, Casiano RR, Shaw GY.

Laryngopharyngeal reflux: position statement of the Committee on Speech, Voice, and Swallowing Disorders of the American Academy of Otolaryngology-Head and Neck Surgery. Otolaryngol Head Neck Surg 2002;127:32-35.

2. Groome M, Cotton JP, Borland M, McLeod S, et al. Prevalence of laryngopharyngeal reflux in a population with gastroesophageal reflux. Laryngoscope 2007;117:1424-1428.

3. Ulualp SO, Rodriguez $\mathrm{S}$, Holmes-Wright CN. Flexible laryngoscopy-guided pharyngeal $\mathrm{pH}$ monitoring in infants. Laryngoscope 2007;117:577-580.

4. Fredrickson JM, Krause CJ, Harker LE, Schuller DE, Richardson MA. Otolaryngology and head and neck surgery. 3rd ed. St. Louis: Mosby; 1999:24112426.

5. Bailey BJ, Johnson JT, Newlands SD, Calhoun KH, Deskin RW. Head and neck surgeryotolaryngology. 4th ed. Philadelphia: Lippincott, 1993;756-761.

6. Ford CN. Evaluation and management of laryngopharyngeal reflux. Rev JAMA 2005;294:1534-1540.

7. Harrell S, Evans B, Goudy S, Winstead W, et al. Design and implementation of an ambulatory $\mathrm{pH}$ monitoring protocol in patients with suspected laryngopharyngeal reflux. Laryngoscope 2005;115(1):8992.

8. Escajadillo JR. Oídos, nariz, garganta y cirugía de cabeza y cuello. $2^{\mathrm{a}}$ ed. Ciudad de México: Editorial El Manual Moderno, 2002;515-518.
9. Fajardo DG, Chavolla MR. Otorrinolaringología. $1^{a}$ ed. Ciudad de México: Inter sistemas, 2003;227-232.

10. Contencin P, Adjoua P, Viala P, Erminy M, Narcy P. [Longterm esophageal and oropharyngeal pH-metry in ORL manifestations of gastroesophageal reflux in children]. Ann Otolaryngol Chir Cervicofac 1992;109(3):129-133

11. Koufman JA, Belafsky PC, Bach KK, Daniel E, Postma GN. Prevalence of esophagitis in patients with $\mathrm{pH}$-documented laryngopharyngeal reflux. Laryngoscope 2002;112(9):16061609

12. Karkos Petros D, Yates Philip D, Carding Paul N, Wilson Janet A. Is Laryngopharyngeal Reflux Related to Functional Dysphonia? Annals of Otology Rhinology \& Laryngology 2007; 116:24-9.

13. Nazar R, Fullá J, Varas M, Naser A. Reflujo faringolaríngeo. Revisión bibliográfica. Revista de otorrinolaringología y cirugía de cabeza y cuello. Santiago 2008; 68:193-198

14. Montes R. Cárdenas R. Incidencia de las manifestaciones otorrinolaringológicas del reflujo gastroesofágico en personas jóvenes de México. Revista de Especialidades Medico quirúrgicas; México 2011; 16(3):152-156.

15. Belafsky PC, Postma GN, Koufman JA, escala de hallazgos de reflujo validez y confiabilidad (RFS). Laringoscopia 2001; 111:1313-1317.

16. 16. Espino E. Los Ángeles Classification of Esophagitis. Gastroenterología latinoamericana 2010; $\mathrm{Vol} 21, \mathrm{~N}^{\circ}$ 2: 184-186. 
Torres López D, Cortadi D, Romero Orellano F Correlación diagnostica entre fibronasolaringoscopía y video endoscopia digestiva alta en pacientes con reflujo gastroesofágico y reflujo faringolaríngeo

\section{ANEXO}

Tabla 1. Índice adaptado de Balafsky.

\begin{tabular}{|l|c|}
\hline \multicolumn{1}{|c|}{ Hallazgos } & Puntaje \\
\hline Pseudosulcus & $0=$ ausente, $2=$ presente \\
\hline Obliteración del ventrículo & $0=$ no hay, $2=$ parcial, $3=$ completa \\
\hline Eritema / hiperemia & $0=$ no hay, $2=$ aritenoides, $3=$ difuso \\
\hline Edema de pliegue vocal & $0=$ no hay, $2=$ leve, $3=$ severo, $4=$ poliploide \\
\hline Edema laríngeo difuso & $0=$ no hay, $2=$ leve, $3=$ severo, $4=$ obstructivo \\
\hline Hipertrofia de la comisura posterior & $0=$ no hay, $2=$ leve, $3=$ severo, $4=$ obstructivo \\
\hline Granuloma / tejido de granulación & $0=$ ausente, $2=$ presente \\
\hline Moco espeso endolaríngeo & $0=$ ausente, $2=$ presente \\
\hline
\end{tabular}

Tabla 2. Clasificación de severidad de esofagitis de Los Ángeles.

\begin{tabular}{|c|l|}
\hline A & Una o más erosiones sobre la mucosa de $5 \mathrm{~mm}$ que no se extienden entre 2 pliegues \\
\hline B & Una o más erosiones $+5 \mathrm{~mm}$ que no se entienden entre 2 pliegues \\
\hline C & $\begin{array}{l}\text { Erosiones que se extienden entre } 2 \text { o más pliegues mucosos, afectando }<75 \% \text { de } \\
\text { circunferencia esofágica }\end{array}$ \\
\hline D & Erosiones que afectan $+75 \%$ de circunferencia esofágica \\
\hline
\end{tabular}

